TITLE:

\title{
Shock structure analysis in chemically reacting gas mixtures by a relaxation-time kinetic model
}

AUTHOR(S):

Groppi, M; Aoki, K; Spiga, G; Tritsch, V

\section{CITATION:}

Groppi, M ... [et al]. Shock structure analysis in chemically reacting gas mixtures by a relaxation-time kinetic model. PHYSICS OF FLUIDS 2008, 20(11): 117103.

\section{ISSUE DATE:}

2008-11

URL:

http://hdl.handle.net/2433/84588

\section{RIGHT:}

Copyright 2008 American Institute of Physics. This article may be downloaded for personal use only. Any other use requires prior permission of the author and the American Institute of Physics. 


\title{
Shock structure analysis in chemically reacting gas mixtures by a relaxation-time kinetic model
}

\author{
M. Groppi, ${ }^{1}$ K. Aoki, ${ }^{2}$ G. Spiga, ${ }^{1, a)}$ and V. Tritsch $^{3}$ \\ ${ }^{1}$ Department of Mathematics, University of Parma, Viale G. P. Usberti 53/A, 43100 Parma, Italy \\ ${ }^{2}$ Department of Mechanical Engineering and Science and Advanced Research Institute of Fluid Science \\ and Engineering, Kyoto University, Kyoto 606-8501, Japan \\ ${ }^{3}$ Department of Mathematics, University of Mainz, Staudingerweg 7, 55128 Mainz, Germany
}

(Received 1 July 2008; accepted 3 October 2008; published online 13 November 2008)

\begin{abstract}
The shock structure in a gas mixture undergoing a bimolecular chemical reaction is studied by means of a reactive kinetic relaxation model. The relevant nonlinear integrodifferential equations are numerically solved in one space dimension with upstream and downstream asymptotic equilibrium conditions satisfying the reactive Rankine-Hugoniot relations and entropy condition. Numerical results are presented, emphasizing the role of Mach number, upstream concentration fractions, and change in the chemical composition across the shock. (C) 2008 American Institute of Physics. [DOI: 10.1063/1.3013637]
\end{abstract}

\section{INTRODUCTION}

Since pioneering times, ${ }^{1}$ bimolecular chemical reactions in a rarefied gas are studied at the kinetic level ${ }^{2}$ on the basis of Boltzmann-like integrodifferential nonlinear equations for gas mixtures, ${ }^{3,4}$ taking into account the additional transfer of mass and energy of chemical bond in each microscopic chemical interaction. ${ }^{5}$ An accurate model requires also a suitable description of nontranslational degrees of freedom, as done recently by allowing either a discrete or a continuous distribution of internal energy levels. ${ }^{6,7}$ The actual kinetic treatment is clearly very heavy from the technical point of view, and therefore the need for reliable simplified models spontaneously arises. In this respect, consistent relaxationtime approximations of the cumbersome Boltzmann collision operators [the so-called Bhatnagar-Gross-Krook (BGK) models] have been proposed for practical and numerical purposes. ${ }^{8-10}$

On one side, the main motivation of a kinetic approach is probably a rigorous derivation of hydrodynamic equations for the macroscopic fields of interest in real world applications. On the other hand, there is a growing interest toward the employment of the kinetic equations themselves for solving classical fundamental problems of fluid dynamics, such as evaporation-condensation, wave propagation, shock structure, and Riemann problem. ${ }^{11}$ The literature is quite rich in this sense for inert gases (see, for instance, Refs. 12-15), but the reactive problems have been typically tackled only at the Euler level (we may quote, among others, Refs. 16-18). To the authors' knowledge, deterministic reactive kinetic computations are restricted up to now to space-homogeneous scenarios $^{19}$ and to the one-dimensional Riemann problem. ${ }^{20}$

This paper is aimed at investigating the steady shock wave problem in a reactive mixture by means of kinetic model equations of relaxation BGK type. Shock wave structure has attracted a lot of attention mainly for the inert prob-

\footnotetext{
a) Author to whom correspondence should be addressed. Electronic mail: giampiero.spiga@unipr.it.
}

lem without chemical reactions and has been deeply studied in the frame of hydrodynamics ${ }^{21}$ and extended thermodynamics. ${ }^{22,23}$ For an analytical study at the kinetic level we shall use here one of the simplest kinetic model (as introduced in Ref. 24 and later enlarged and improved in Ref. 25), which, in spite of its simplifications, has been shown to capture the essential features of the chemical interaction. The main properties of the model are recalled below for the readers' convenience in view of its specific application to the shock problem. The Rankine-Hugoniot conditions following from the conservation laws and the entropy condition induced by Boltzmann's lemma have been derived and discussed for the reactive problem in a recent paper $^{26}$ and will provide the physical frame in which our BGK calculations are cast. The BGK model equations to be used here for the reactive collision operator originate from an idea in Ref. 27, where Andries et al. introduced a consistent approximation of kinetic equations for (inert) gas mixtures, in which all typical drawbacks encountered by BGK models for more than one species (such as breakdown of positivity and indifferentiability) were overcome. Numerical results are presented and discussed for various parameter sets, in particular for varying Mach numbers, upstream conditions, and reaction rates.

\section{KINETIC APPROACH TO THE REACTIVE SHOCK PROBLEM}

A four component gas mixture of interacting species $A^{i}$, $i=1, \ldots, 4$, undergoing the reversible bimolecular chemical reaction

$$
A^{1}+A^{2} \rightleftharpoons A^{3}+A^{4},
$$

as well as binary elastic collisions, may be described at the kinetic level, according to the model proposed in Ref. 24, and then generalized to reacting species with internal structure in Ref. 6 by the set of nonlinear integrodifferential Boltzmann-like equations 


$$
\frac{\partial f^{i}}{\partial t}+\mathbf{v} \cdot \frac{\partial f^{i}}{\partial \mathbf{x}}=Q^{i}[f], \quad i=1, \ldots, 4,
$$

where $f$ is the vector of the four distribution functions $f^{i}(\mathbf{x}, \mathbf{v}, \bar{t})$. Nontranslational degrees of freedom are neglected here for simplicity. The collision terms $Q^{i}$ may be split into their mechanical and chemical parts, accounting for the proper conservation of mass, momentum, and energy, where the latter involves also the bond energies $E^{i}$, with energy gap

$$
\Delta E=-\sum_{i=1}^{4} \Lambda^{i} E^{i}=E^{3}+E^{4}-E^{1}-E^{2},
$$

where $\Lambda^{i}$ is a component of the string of the stoichiometric coefficients $(1,1,-1,-1)$. Species may always be ordered in such a way that the sign of $\Delta E$ is positive so that we will stick to such option, without any loss of generality. Collision invariants constitute a seven-dimensional linear subspace in the space of the continuous functions of $\mathbf{v}$ and represent conservation of momentum, total (thermal plus chemical) internal energy, and particles in the independent pairs of species $(1,3),(1,4)$, and $(2,4)$. Referring the interested reader to the quoted bibliography (see, for instance, Ref. 25 for a review), the global collision term $Q$ obeys an extended version of Boltzmann's lemma in terms of a suitable Lyapunov functional, and collision equilibria are identified as the seven parameter family of local Maxwellians,

$f_{M}^{i}(\mathbf{v})=n^{i}\left(\frac{m^{i}}{2 \pi K T}\right)^{3 / 2} \exp \left[-\frac{m^{i}}{2 K T}(\mathbf{v}-\mathbf{u})^{2}\right], \quad i=1, \ldots, 4$,

with $\mathbf{u}$ and $T$ standing for mass velocity and temperature of the mixture, respectively. Here $m^{i}$ denotes the mass of the $i$ th species and $K$ is the Boltzmann constant. In addition, on account of microreversibility, number densities $n^{i}$ must be related by the law of mass action,

$$
\frac{n^{1} n^{2}}{n^{3} n^{4}}=\left(\frac{\mu^{12}}{\mu^{34}}\right)^{3 / 2} \exp \left(\frac{\Delta E}{K T}\right),
$$

in which $\mu^{i j}$ stands for the reduced mass $m^{i} m^{j} /\left(m^{i}+m^{j}\right)$. One might also introduce the concentration fractions

$$
\chi^{i}=\frac{n^{i}}{n}, \quad \sum_{i=1}^{4} \chi^{i}=1
$$

and notice that Eq. (5) may be rewritten in equivalent forms, like, for instance,

$\frac{\chi^{1} \chi^{2}}{\chi^{3} \chi^{4}}=\eta \exp \left(\frac{\Delta E}{K T}\right) \quad$ or $\frac{K T}{\Delta E}=\left[\sum_{i=1}^{4} \Lambda^{i} \log \frac{\chi^{i}}{\left(m^{i}\right)^{3 / 2}}\right]^{-1}$,

where the parameter $\eta$ is defined by

$$
\eta=\left(\frac{m^{1} m^{2}}{m^{3} m^{4}}\right)^{3 / 2}
$$

and turns out to be different from unity if an actual reaction takes place. Entropy density $s$ at local thermodynamical equilibrium, proportional to the pertinent $H$-functional ${ }^{24}$ evaluated at the Maxwellian state (4), may be defined as

$$
s=-K \sum_{i=1}^{4} \chi^{i} \log \frac{\chi^{i}}{\left(m^{i}\right)^{3 / 2}}+\frac{3}{2} K \log T-K \log n,
$$

where the last two addends make up formally the standard single gas entropy, and bearing the mass action law (7) in mind, it depends only on four macroscopic fields. The simplest approximate hydrodynamic limit is provided as usual by the Euler equations, which are, in a regime dominated by any kind of collisions (mechanical and chemical, thus a fast process), a set of seven hyperbolic partial differential equations to be coupled with the mass action law (5) to provide a set of eight differential-algebraic equations for the eight unknown functions $n^{i}, \mathbf{u}, T$. A standard procedure allows then to deduce the speed of sound for the considered reactive mixture ${ }^{17,18}$

$$
\begin{aligned}
c_{0}= & \left\{\frac{5}{3} \frac{n K T}{\rho}\left[\sum_{i=1}^{4} \frac{1}{\chi^{i}}+\frac{2}{5}\left(\frac{\Delta E}{K T}\right)^{2}\right] /\right. \\
& {\left.\left[\sum_{i=1}^{4} \frac{1}{\chi^{i}}+\frac{2}{3}\left(\frac{\Delta E}{K T}\right)^{2}\right]\right\}^{1 / 2}, }
\end{aligned}
$$

which shows a deviation from the standard value for the inert mixture, given by $\sqrt{(5 / 3)(n K T / \rho)}$, determined by chemical composition alone since temperature $T$ may be eliminated via Eq. (5); in this sense, it is independent of the jump $\Delta E$ in the energies of chemical bonds. The deviation is always a reduction in the inert sound speed, but anyway the reactive sound speed can never decrease beyond the lower bound $\sqrt{n K T / \rho}$.

The steady shock problem in one space dimension may be formulated as follows. ${ }^{11}$ Consider the kinetic equations in the simple stationary form,

$$
v_{1} \frac{\partial f^{i}}{\partial x}=Q^{i}[f], \quad i=1, \ldots, 4,
$$

with $v_{1}$ standing for the first component of the velocity vector $\mathbf{v}$ and with $x=x_{1}$. Equation (11) has to be solved for $-\infty<x<+\infty$ with boundary conditions,

$$
\lim _{x \rightarrow \pm \infty} f^{i}(x, \mathbf{v})=M_{ \pm}^{i}(\mathbf{v}),
$$

where $M_{ \pm}^{i}$ denotes families of Maxwellian distributions describing equilibrium outside the shock region, characterized by upstream (-) and downstream (+) parameters $n_{ \pm}^{i}, u_{ \pm}$ $=u_{1 \pm}$, and $T_{ \pm}$. Temperatures are not independent but follow from the mass action laws,

$$
\frac{\chi_{ \pm}^{1} \chi_{ \pm}^{2}}{\chi_{ \pm}^{3} \chi_{ \pm}^{4}}=\eta \exp \left(\frac{\Delta E}{K T_{ \pm}}\right),
$$

where, since $\Delta E>0$, the following constraint

$$
\frac{\chi_{ \pm \pm}^{1} \chi_{ \pm}^{2}}{\chi_{ \pm}^{3} \chi_{ \pm}^{4}}>\eta
$$

must be in order. We recall here for the readers' convenience some of the main conclusions of the detailed analysis that can be found in Ref. 26. The ten free upstream and downstream parameters are related by the Rankine-Hugoniot con- 
ditions, which simply express the five conservation laws holding for the present problem in one space dimension and allow, in principle, to deduce the five downstream parameters in terms of the set of the corresponding upstream ones. Skipping all details, the procedure of integrating on the real line the conservation equations for the appropriate fluxes and applying limiting condition (12) leads to a set of five nonlinear algebraic equations that can be solved analytically upon introducing $\Delta \chi=\Delta \chi^{1}$ as independent variable, where $\Delta \chi^{i}$ $=\chi_{+}^{i}-\chi_{-}^{i}$. A first result is that

$$
\chi_{+}^{i}=\chi_{-}^{i}+\Lambda^{i} \Delta \chi, \quad i=1, \ldots, 4, \quad \frac{n_{+}}{n_{-}}=\frac{u_{-}}{u_{+}}=\frac{\rho_{+}}{\rho_{-}},
$$

with, in particular, $\Delta \chi^{1}=\Delta \chi^{2}=-\Delta \chi^{3}=-\Delta \chi^{4}$. In addition, we get a unique meaningful solution for the ratio $n_{+} / n_{-}$, namely,

$$
\begin{aligned}
\frac{n_{+}}{n_{-}}= & 2\left(1-\frac{T_{-}}{T_{+}}\right)-\frac{\Delta E}{K T_{+}} \Delta \chi \\
& +\sqrt{\left[2\left(1-\frac{T_{-}}{T_{+}}\right)-\frac{\Delta E}{K T_{+}} \Delta \chi\right]^{2}+\frac{T_{-}}{T_{+}}} .
\end{aligned}
$$

Moreover, assuming conventionally $u_{-}>0$ ( $u$ can never cross the value zero in the shock), we have then

$$
u_{-}=\sqrt{\frac{n_{-} K T_{-}}{\rho_{-}}}\left[\frac{n_{+}}{n_{-}} \frac{1-\left(n_{+} / n_{-}\right)\left(T_{+} / T_{-}\right)}{1-\left(n_{+} / n_{-}\right)}\right]^{1 / 2}
$$

provided that

$$
\left(1-\frac{n_{+}}{n_{-}} \frac{T_{+}}{T_{-}}\right)\left(1-\frac{n_{+}}{n_{-}}\right)>0 .
$$

Finally, from Eq. (15), we have $u_{+}=u_{-} /\left(n_{+} / n_{-}\right)$, which completes the procedure and determines uniquely all downstream parameters and $u_{-}$in terms of $n_{-}^{i}$ and $\Delta \chi$.

The other crucial physical feature of the shock wave problem, which follows from the mathematical properties of the collision operator, is related to the second law of thermodynamics. This leads to a differential inequality for the entropy flux that amounts to the requirement, $\Delta s=s_{+}-s_{-} \geq 0$, of clear physical meaning. However, its solution is much harder than for the corresponding investigation relevant to the case of a nonreacting gas, although again it can be performed in terms of the upstream Mach number Ma_. The Mach numbers in the limiting equilibrium states are defined as usual by

$$
\mathrm{Ma}_{ \pm}=u_{ \pm} / c_{0 \pm},
$$

with $c_{0}$ denoting the up- or downstream sound speed provided in the present reactive case by expression (10).

The analytical and numerical investigation in Ref. 26 leads to the result that the above entropy condition is fulfilled exactly under the same condition holding in the inert case, namely,

$$
\mathrm{Ma}_{-} \geq 1,
$$

implying that only transitions from a supersonic to a subsonic flow have a physical meaning. Indeed, strict inequality is actually required since equal sign would imply identical states at $\pm \infty$. Moreover, and remarkably enough, concerning the change in the chemical composition that definitely must take place for the reactive problem, the variation always goes in a well defined direction characterized by $\Delta \chi<0$ (actually, there is a one-to-one correspondence between $\mathrm{Ma}_{-}$and $\Delta \chi$, the latter being negative or positive according to whether the wave front is supersonic or subsonic). For the shock process, this necessary and sufficient condition on the sign of $\Delta \chi$ corresponds then to an enhancement of the products at the expense of the reactants (macroscopically endothermic shift). The downstream chemical equilibrium carries then a larger chemical energy $(\Delta E>0, \Delta \chi<0)$ as well as a larger thermal energy $\left[T_{+}>T_{-}\right.$as follows from Eq. (7) and from $\left.\Delta \chi<0\right]$. More explicitly, the constraint on $\Delta \chi$ reads as

$$
-\chi_{-}^{*}<\Delta \chi<0,
$$

where, for any $\eta \neq 1$,

$$
\begin{aligned}
\chi_{-}^{*}= & \frac{1}{2(1-\eta)}\left\{\chi_{-}^{1}+\chi_{-}^{2}+\eta \chi_{-}^{3}+\eta \chi_{-}^{4}\right. \\
& \left.-\sqrt{\left(\chi_{-}^{1}+\chi_{-}^{2}+\eta \chi_{-}^{3}+\eta \chi_{-}^{4}\right)^{2}-4(1-\eta)\left(\chi_{-}^{1} \chi_{-}^{2}-\eta \chi_{-}^{3} \chi_{-}^{4}\right)}\right\}
\end{aligned}
$$

is determined from boundedness and positivity requirements for the macroscopic fields; in particular, $\chi_{-}^{*}<\min \left\{\chi_{-}^{1}, \chi_{-}^{2}\right\}$. In the frame outlined in this section, we are going now to proceed to the actual calculation of the shock profile by solving Eqs. (11) in their relaxation-time approximation.

\section{BGK MODEL EQUATIONS}

The relaxation model used here for the reactive collision operator $Q$ of the present problem was proposed first in Ref. 9 for space-homogeneous calculations. Following a clever idea of Andries et al. ${ }^{27}$ the underlying strategy is very simple and consists in introducing only one BGK operator for each species, accounting for any type of interaction with whatever other species. We refer the interested reader to the papers quoted above for technical details and deeper discussion. We just mention here that the model is constructed in such a way that the $i$ th BGK operator drifts the distribution function $f^{i}$ toward a suitable Gaussian function $\mathcal{M}_{i}$ with fictitious macroscopic parameters $n_{i}, \mathbf{u}_{i}$, and $T_{i}$ (different from the actual ones, $n^{i}, \mathbf{u}^{\mathrm{i}}$, and $T^{i}$ ), and such parameters are chosen in such a way that the exact exchange rates for mass, momentum, and energy of each species are correctly reproduced. Using such a philosophy, it has been possible to prove that BGK equations retain most of the significant features of the original kinetic equations. For instance, they preserve the correct collision invariants, conservation equations, and local equilibria (4) with mass action law (5). Also, in the inert case, it has been possible to prove explicitly an $H$-theorem. Such a result has not been achieved yet for the reactive case but it has been verified numerically to hold for the actual $H$-functional. The key point for the practical realization of this BGK approximation, which looks promising a priori and has proved satisfactory in several homogeneous and spacedependent applications, ${ }^{19,20}$ is the availability of the exact Boltzmann exchange rates for mass, momentum, and energy. They are known in closed analytical form for the elastic scat- 
tering operator in the collision model of Maxwellian molecules, so that we will stick for simplicity also here to this common option. The situation is even more complicated for the chemical collision integrals. Unless input distribution functions are suitably approximated a priori (see, for instance, Ref. 10 for a computation of elastic and reactive exchange rates relevant to different types of cross sections), analytical exchange rates have been evaluated, to our knowledge, only in Ref. 28 under an assumption equivalent to Maxwellian molecules. Specifically, the microscopic collision frequency for endothermic reaction was taken to be constant versus the relative speed on its support in a physical regime in which mechanical relaxation is significantly faster than the chemical one. Although these restrictions are quite strong (negligible activation energy and slow chemical reactions only), we will adopt them here for this first nonmacroscopic approach to the reactive shock wave problem.

Model equations for our reactive mixture in one space dimension take the form

$$
v_{1} \frac{\partial f^{i}}{\partial x}=\bar{Q}^{i}[f]=\nu_{i}\left(\mathcal{M}_{i}-f^{i}\right), \quad i=1, \ldots, 4,
$$

where

$\mathcal{M}_{i}=n_{i}\left(\frac{m^{i}}{2 \pi K T_{i}}\right)^{3 / 2} \exp \left[-\frac{m^{i}}{2 K T_{i}}\left(\mathbf{v}-\mathbf{u}_{i}\right)^{2}\right], \quad i=1, \ldots, 4$

and $\nu_{i}$ are suitable $\mathbf{v}$-independent (macroscopic) collision frequencies, defined later. All collision parameters $\nu_{k}^{i j}$ and $\nu_{12}^{34}$ needed in the sequel, which represent suitable angular integrations of the microscopic (mechanical and chemical) collision frequencies, are constant in our assumptions (see Ref. 9 for details). The exchange rates underlying the ansatz (23) are

$$
\begin{aligned}
& \int_{\mathbb{R}^{3}} \bar{Q}^{i} d_{3} \mathbf{v}=\nu_{i}\left(n_{i}-n^{i}\right), \\
& \int_{\mathbb{R}^{3}} m^{i} \mathbf{v} \bar{Q}_{i} d_{3} \mathbf{v}=\nu_{i} m^{i}\left(n_{i} \mathbf{u}_{i}-n^{i} \mathbf{u}^{i}\right), \\
& \int_{\mathbb{R}^{3}} \frac{m^{i}}{2} v^{2} \bar{Q}_{i} d_{3} \mathbf{v} \\
& =\nu_{i}\left[n_{i} \frac{3}{2} K T_{i}-n^{i} \frac{3}{2} K T^{i}+n_{i} \frac{1}{2} m^{i} u_{i}^{2}-n^{i} \frac{1}{2} m^{i}\left(u^{i}\right)^{2}\right] .
\end{aligned}
$$

Under the hypotheses mentioned above, the same exchange rates for the actual mechanical $\left(I^{i}=\sum_{j=1}^{4} I^{i j}\right)$ and chemical $\left(J^{i}\right)$ collision operators are

$$
\begin{aligned}
& \int_{\mathbb{R}^{3}} I^{i} d_{3} \mathbf{v}=0, \\
& \int_{\mathbb{R}^{3}} m^{i} \mathbf{v} I^{i} d_{3} \mathbf{v}=\sum_{j=1}^{4} \nu_{1}^{i j} \mu^{i j} n^{i} n^{j}\left(\mathbf{u}^{j}-\mathbf{u}^{i}\right),
\end{aligned}
$$

$$
\begin{aligned}
\int_{\mathbb{R}^{3}} \frac{m^{i}}{2} v^{2} I^{i} d_{3} \mathbf{v}= & \sum_{j=1}^{4} \nu_{1}^{i j} \frac{2 \mu^{i j}}{m^{i}+m^{j}} n^{i} n^{j}\left[\frac{3}{2} K\left(T^{j}-T^{i}\right)\right. \\
& \left.+\frac{1}{2}\left(m^{j} \mathbf{u}^{j}+m^{i} \mathbf{u}^{i}\right) \cdot\left(\mathbf{u}^{j}-\mathbf{u}^{i}\right)\right],
\end{aligned}
$$

and

$$
\begin{aligned}
\int_{\mathrm{R}^{3}} J^{i} d_{3} \mathbf{v}=\Lambda^{i} \mathcal{S}, & \int_{\mathbb{R}^{3}} m^{i} \mathbf{v} J^{i} d_{3} \mathbf{v}=\Lambda^{i} \mathcal{S} m^{i} \mathbf{u} \\
\int_{\mathrm{R}^{3}} \frac{m^{i}}{2} v^{2} J^{i} d_{3} \mathbf{v}= & \Lambda^{i} \mathcal{S}\left\{\frac{1}{2} m^{i} u^{2}+\frac{3}{2}(K T)-\frac{1-\Lambda^{i}}{2} \frac{M-m^{i}}{M} \Delta E\right. \\
& +\frac{M-m^{i}}{M} K T\left(\frac{\Delta E}{K T}\right)^{3 / 2} \\
& \left.\times \exp \left(-\frac{\Delta E}{K T}\right)\left[\Gamma\left(\frac{3}{2}, \frac{\Delta E}{K T}\right)\right]^{-1}\right\}
\end{aligned}
$$

where $M$ is the total mass $\left(M=m^{1}+m^{2}=m^{3}+m^{4}\right)$ and

$$
\mathcal{S}=\nu_{12}^{34} \frac{2}{\sqrt{\pi}} \Gamma\left(\frac{3}{2}, \frac{\Delta E}{K T}\right)\left[n^{3} n^{4}\left(\frac{m^{1} m^{2}}{m^{3} m^{4}}\right)^{3 / 2} \exp \left(\frac{\Delta E}{K T}\right)-n^{1} n^{2}\right],
$$

with $\Gamma$ denoting an incomplete gamma function. ${ }^{29}$ Equating the global BGK rates (25) to the sum of the mechanical rates (26) and the chemical rates (27) allows one to determine explicitly the 20 auxiliary parameters $n_{i}, \mathbf{u}_{i}, T_{i}$ in terms of the actual fields, as

$$
\begin{aligned}
n_{i}=n^{i}+ & \frac{\Lambda^{i}}{\nu_{i}} \mathcal{S}, \quad m^{i} n_{i} \mathbf{u}_{i}=m^{i} n^{i} \mathbf{u}^{i}+\frac{1}{\nu_{i j}} \sum_{j=1}^{4} \phi^{i j} \mathbf{u}^{j}+\frac{\Lambda^{i}}{\nu_{i}} m^{i} \mathbf{u} \mathcal{S}, \\
n_{i} \frac{3}{2} K T_{i}= & n^{i} \frac{3}{2} K T^{i}-\frac{1}{2} m^{i}\left[n_{i} u_{i}^{2}-n^{i}\left(u^{i}\right)^{2}\right]+\frac{1}{\nu_{i j}} \sum_{j=1}^{4} \psi^{i j} T^{j} \\
& +\frac{1}{\nu_{i}} \sum_{j=1}^{4} \nu_{1}^{i j} \frac{\mu^{i j}}{m^{i}+m^{j}} n^{i} n^{j}\left(m^{i} \mathbf{u}^{i}+m^{j} \mathbf{u}^{j}\right) \cdot\left(\mathbf{u}^{j}-\mathbf{u}^{i}\right) \\
& +\frac{\Lambda^{i}}{\nu_{i}} \mathcal{S}\left\{\frac{1}{2} m^{i} u^{2}+\frac{3}{2} K T+\frac{M-m^{i}}{M} K T\left(\frac{\Delta E}{K T}\right)^{3 / 2}\right. \\
& \left.\times \frac{\exp \left(-\frac{\Delta E}{K T}\right)}{\Gamma\left(\frac{3}{2}, \frac{\Delta E}{K T}\right)}-\frac{1-\Lambda^{i}}{2} \frac{M-M^{i}}{M} \Delta E\right\} .
\end{aligned}
$$

We refer again to Ref. 9 for the definition of matrices $\left(\phi^{i j}\right)$ and $\left(\psi^{i j}\right)$, as well as for the choice of the inverse collision times $\nu_{i}$, for which an estimate of the number of collisions taking place in the mixture suggests the expression 


$$
\begin{aligned}
& \nu_{1}=\sum_{j=1}^{4} \nu_{0}^{1 j} n^{j}+\frac{2}{\sqrt{\pi}} \Gamma\left(\frac{3}{2}, \frac{\Delta E}{K T}\right) \nu_{12}^{34} n^{2}, \\
& \nu_{2}=\sum_{j=1}^{4} \nu_{0}^{2 j} n^{j}+\frac{2}{\sqrt{\pi}} \Gamma\left(\frac{3}{2}, \frac{\Delta E}{K T}\right) \nu_{12}^{34} n^{1}, \\
& \nu_{3}=\sum_{j=1} \nu_{0}^{3 j} n^{j}+\frac{2}{\sqrt{\pi}} \Gamma\left(\frac{3}{2}, \frac{\Delta E}{K T}\right)\left(\frac{\mu^{12}}{\mu^{34}}\right)^{3 / 2} \exp \left(\frac{\Delta E}{K T}\right) \nu_{12}^{34} n^{4}, \\
& \nu_{4}=\sum_{j=1}^{4} \nu_{0}^{4 j} n^{j}+\frac{2}{\sqrt{\pi}} \Gamma\left(\frac{3}{2}, \frac{\Delta E}{K T}\right)\left(\frac{\mu^{12}}{\mu^{34}}\right)^{3 / 2} \exp \left(\frac{\Delta E}{K T}\right) \nu_{12}^{34} n^{3} .
\end{aligned}
$$

As usual, it is worth to devise a suitable scaling in order to make governing equations dimensionless. For a given reaction, the jump $\Delta E$ is fixed and one can identify typical values $\nu_{*}$ for the microscopic collision frequencies $\left(\nu_{0}^{i j}, \nu_{1}^{i j}, \nu_{12}^{34}\right)$ and $m_{*}$ for masses. One can also determine a typical density $n_{*}$. This suggests resorting to the typical collision time $\tau$, speed $\xi$, and length $l$ defined by

$$
\tau=\frac{1}{\nu_{*} n_{*}}, \quad \xi=\left(\frac{\Delta E}{m_{*}}\right)^{1 / 2}, \quad l=\xi \tau .
$$

The adimensionalization we are going to use in order to make results as universal as possible is defined by

$$
\begin{aligned}
& m^{i}=m_{*} \widetilde{m}^{i}, \quad \nu_{i}=\nu_{*} n_{*} \widetilde{\nu}_{i}, \quad \nu_{k}^{i j}=\nu_{*} \widetilde{\nu}_{k}^{i j}, \\
& \nu_{12}^{34}=\nu_{*} \widetilde{\nu}_{12}^{34}, \quad f^{i}=\frac{n_{*}}{\xi^{3}} \widetilde{f}^{i},
\end{aligned}
$$

which implies

$$
\begin{aligned}
& n^{i}=n_{*} \widetilde{n}^{i}, \quad \mathbf{u}^{i}=\xi \widetilde{\mathbf{u}}^{i}, \quad T^{i}=\frac{\Delta E}{K} \widetilde{T}^{i}, \\
& n_{i}=n_{*} \widetilde{n}_{i}, \quad \mathbf{u}_{i}=\xi \widetilde{\mathbf{u}}_{i}, \quad T_{i}=\frac{\Delta E}{K} \widetilde{T}_{i} .
\end{aligned}
$$

Dimensionless equations would be

$$
\widetilde{v}_{1} \frac{\partial \tilde{f}^{i}}{\partial \tilde{x}}=\widetilde{v}_{i}\left(\tilde{\mathcal{M}}_{i}-\tilde{f}^{i}\right), \quad i=1, \ldots, 4,
$$

where

$$
\begin{aligned}
& \tilde{\mathcal{M}}_{i}=\tilde{n}_{i}\left(\frac{\tilde{m}^{i}}{2 \pi \widetilde{T}_{i}}\right)^{3 / 2} \exp \left[-\frac{\tilde{m}^{i}}{2 \widetilde{T}_{i}}\left(\widetilde{\mathbf{v}}-\widetilde{\mathbf{u}}_{i}\right)^{2}\right], \quad i=1, \ldots, 4, \\
& \widetilde{\nu}_{1}=\sum_{j=1}^{4} \widetilde{\nu}_{0}^{1 j} \widetilde{n}^{j}+\frac{2}{\sqrt{\pi}} \Gamma\left(\frac{3}{2}, \frac{1}{\widetilde{T}}\right) \widetilde{\nu}_{12}^{34} \widetilde{n}^{2},
\end{aligned}
$$

and so on. Therefore, if all tildes are then removed, equations read the same as the dimensional ones, apart from the scalings. In particular, $\Delta E$, which, for a given reaction, is determined once for all and cannot play the role of a free parameter, is normalized to unity, and the dimensionless mass action law reads as

$$
\frac{\chi^{1} \chi^{2}}{\chi^{3} \chi^{4}}=\left(\frac{m^{1} m^{2}}{m^{3} m^{4}}\right)^{3 / 2} \exp \left(\frac{1}{T}\right)
$$

Now, contrary to the standard procedure, $l$ is not related to any mean free path. A mean free path could be rather introduced if the scaling was performed in terms of the macroscopic quantities assigned as boundary conditions. However the advantage here is that $l$ does not change when essential control parameters of the problem such as the upstream chemical composition are varied, and therefore the relevant results can be compared directly on a common scale. However, mean free paths do affect numerical computations, especially the size of the finite space and velocity domains of integration, so attention must be paid in order to ensure numerical accuracy.

\section{RESULTS AND DISCUSSION}

We show explicitly in this section the shock profiles resulting from the numerical integration of the dimensionless BGK model equations worked out in Sec. III for various choices of parameters, essentially the concentration change $\Delta \chi$ (equivalent to Mach number $\mathrm{Ma}_{-}$), upstream chemical composition, and reactive collision frequency.

\section{A. Numerical implementation}

Following the idea of $\mathrm{Chu}$ et al., ${ }^{30}$ we can eliminate velocity components $v_{2}, v_{3}$ perpendicular to the flow direction $x$ by multiplying Eqs. (34) and (35) by 1 and $v_{2}^{2}+v_{3}^{2}$ and integrating over the whole space $\mathbb{R}^{2}$. This procedure leads to the introduction of new unknowns depending only on $(x, v$ $\left.=v_{1}\right)$,

$$
\begin{aligned}
& \phi_{1}^{i}(x, v)=\int_{\mathbb{R}^{2}} f^{i} \mathrm{~d} v_{2} \mathrm{~d} v_{3}, \\
& \phi_{2}^{i}(x, v)=\int_{\mathbb{R}^{2}}\left(v_{2}^{2}+v_{3}^{2}\right) f^{i} \mathrm{~d} v_{2} \mathrm{~d} v_{3},
\end{aligned}
$$

and allows one to deduce from Eqs. (34) and (35) the following governing equations for the vector function $\underline{\phi}^{i}=\left(\phi_{1}^{i}, \phi_{2}^{i}\right)^{\top}$ :

$v \frac{\partial \underline{\phi}^{i}}{\partial x}=\nu_{i}\left(\underline{\mathcal{F}}^{i}-\underline{\phi}^{i}\right), \quad x \in \mathbb{R}, \quad v \in \mathbb{R}, \quad i=1, \ldots, 4$,

where

$$
\begin{aligned}
& \underline{\mathcal{F}}^{i}=\left(M_{i}, \frac{2 T_{i}}{m^{i}} M_{i}\right)^{\top}, \\
& M_{i}=n_{i}\left(\frac{m^{i}}{2 \pi T_{i}}\right)^{1 / 2} \exp \left[-\frac{m^{i}}{2 T_{i}}\left(v-u_{i}\right)^{2}\right] .
\end{aligned}
$$

In this new formulation, macroscopic moments of distribution functions $f^{i}$ are then given in terms of $\underline{\phi}^{i}$ as 


$$
\begin{aligned}
& n^{i}=\int_{\mathbb{R}} \phi_{1}^{i} \mathrm{~d} v, \quad u^{i}=\frac{1}{n^{i}} \int_{\mathbb{R}} v \phi_{1}^{i} \mathrm{~d} v, \\
& \frac{3 T^{i}}{m^{i}}=\frac{1}{n^{i}}\left[\int_{\mathbb{R}}\left(v-u^{i}\right)^{2} \phi_{1}^{i} \mathrm{~d} v+\int_{\mathbb{R}} \phi_{2}^{i} \mathrm{~d} v\right]
\end{aligned}
$$

(where $u^{i}=u_{1}^{i}$, being $u_{2}^{i}=u_{3}^{i}=0$ ).

Problems (39)-(41) are then discretized as follows. Consider a suitably large finite domain $S_{x} \times S_{v}$, where $S_{x}=[-D, D]$ and $S_{v}=[-V, V]$. Grid points in the domain $S_{x}$ are denoted by $x_{j}$, with $j=-N_{x}, \ldots, N_{x}$ and $\Delta x_{j}=x_{j}-x_{j-1}$; grid points in the domain $S_{v}$ are denoted by $v_{l}, l=-N_{v}, \ldots, N_{v}$ with uniform mesh size $\Delta v$.

Macroscopic moments (42) are numerically computed by integrating over the velocity domain $[-V, V]$ using the composite Simpson rule of order $\mathcal{O}\left(\Delta v^{4}\right)$. Boundary conditions for $\phi^{i}$ at $x= \pm D$ are obtained from the Maxwellians $M_{ \pm}^{i}$ characterized by upstream (-) and downstream (+) parameters related by the Rankine-Hugoniot conditions discussed in Sec. II.

Let $\underline{\phi}_{j, l}^{i}=\underline{\phi}^{i}\left(x_{j}, v_{l}\right), j=-N_{x}, \ldots, N_{x}, \quad l=-N_{v}, \ldots, N_{v}$ be the discretized numerical solution of Eqs. (39)-(41); it is determined by means of the following iterative scheme, first order in space, based on upwind finite difference approximation: ${ }^{31}$ for $k=0,1, \ldots$,

(i) for $l=1, \ldots, N_{v}$ (namely, $\left.v_{l}>0\right)$ and $j=-N_{x}+1, \ldots$, $N_{x}-1$

$$
v_{l} \frac{\underline{\phi}_{j, l}^{i(k+1)}-\underline{\phi}_{j-1, l}^{i(k+1)}}{\Delta x_{j}}=\nu_{i ; j}^{(k)}\left(\underline{\mathcal{F}}_{j, l}^{i(k)}-\underline{\phi}_{j, l}^{i(k+1)}\right),
$$

(ii) for $l=-N_{v}, \ldots, 0$ (namely, $v_{l} \leq 0$ ) and $j=N_{x}-1, \ldots$, $-N_{x}+1$

$$
v_{l} \frac{\underline{\phi}_{j+1, l}^{i(k+1)}-\underline{\phi}_{j, l}^{i(k+1)}}{\Delta x_{j+1}}=\nu_{i ; j}^{(k)}\left(\underline{\mathcal{F}}_{j, l}^{i(k)}-\underline{\phi}_{j, l}^{i(k+1)}\right) .
$$

The iterative procedure on index $k$ is initialized by assigning the profile $\phi^{i(0)}(x, v)$ in the grid points; such initial profile has been chosen as a step function of $v$ connecting the upstream and downstream uniform states. The procedure on $k$ continues until a convergence check is fulfilled. More precisely, every tenth $k$ steps the variation at point $x_{j}$ of the global moments $\left(n_{j}^{(k)}, u_{j}^{(k)}, T_{j}^{(k)}\right)$ during ten steps is summed up; then the maximum over all $j \in\left\{-N_{x}, N_{x}\right\}$ of each of these three values is taken, $(\Delta n, \Delta u, \Delta T)$; the iterative procedure stops when the $L^{\infty}$ norm of this vector is less than $10^{-6}$ (for more details about the numerical procedure see Ref. 32).

A crucial point of the calculation is the correct choice of the computational domains, namely, of the constants $D$ and $V$. As is well known, these constants have to be large enough so that the deviation of the distribution functions from the upstream (downstream) Maxwellians around $x=-D(x=D)$ is negligibly small or smaller than the error of the numerical computation. On the other hand, discretized shock wave solution is affected by the so-called "shift phenomenon," 12 namely, a small but almost constant shift of the shock profiles in each iteration due to the fact that Rankine-Hugoniot conditions cannot be satisfied exactly because of the compu- tational error. For these reasons, the value of $D$ has been chosen larger and larger in order to reduce the shift error, improving then the accuracy of the results, and also to match the convergence check in a reasonable number of iterations.

\section{B. Examples and comments}

As reference reaction we take $\mathrm{NO}+\mathrm{CO}_{2} \rightleftharpoons \mathrm{NO}_{2}+\mathrm{CO}$, corresponding to (dimensionless) masses

$m^{1}=1.0000, \quad m^{2}=1.4667, \quad m^{3}=1.5332, \quad m^{4}=0.9335$,

with $\eta=1.0374>1$; for this reaction we have $\Delta E=3.749$ $\times 10^{-22} \mathrm{~kJ}[225.79 \mathrm{~kJ} / \mathrm{mol}$ for standard enthalpy (see, for instance, NIST Chemical Kinetics Database on the Web)]. Results presented in this paper will be restricted to it for brevity since the main qualitative features do not change appreciably for all several other reactions that have been tested. Also, we need to assign, for fixed $s=0,1$, the scattering collision matrices $\nu_{s}^{i j}, 1 \leq i, j \leq 4$, and for simplicity all of their entries will be set here, once for all, equal to unity. In order to comply with the features of the proposed model, the reactive collision frequency will take quite smaller values. The reference test case (to be labeled as problem A) is relevant to the options $\nu_{12}^{34}=0.03, n_{-}=1$, and to the following upstream concentration fractions:

$$
\chi_{-}^{1}=0.25, \quad \chi_{-}^{2}=0.35, \quad \chi_{-}^{3}=0.25, \quad \chi_{-}^{4}=0.15,
$$

implying $T_{-}=1.2337$. We select then for the numerical implementation of our reactive BGK equations the following changes in the chemical composition (or, equivalently, the Mach numbers corresponding to them):

$$
\begin{aligned}
& \Delta \chi=-0.03,-0.01,-0.001 \\
& \left(\mathrm{Ma}_{-}=2.4698,1.2856,1.0227\right) .
\end{aligned}
$$

The other asymptotic boundary values determined by the Rankine-Hugoniot conditions are

$$
\begin{aligned}
& u_{-}=3.1069,1.6172,1.2865, \\
& u_{+}=1.1565,1.1373,1.2441, \\
& n_{+}=2.6864,1.4219,1.0341, \\
& T_{+}=3.3614,1.5698,1.2609 .
\end{aligned}
$$

All profiles are obtained through Eq. (42) with $\phi^{i}$ replaced by its discretized representation resulting from Eqs. (43) and (44). Computational parameters $D, V, N_{x}$, and $N_{v}$ have been properly chosen in each specific test in order to ensure accuracy of the results, as discussed above. In the presented calculations, it has been sufficient to take $N_{x}=1500, N_{v}=300$, with $V$ ranging from 20 to 50 and $D$ from 500 (for the largest values of $\nu_{12}^{34}$ ) up to 5000 (for the smallest values of the same parameter). In the figures shown here, the $x$ domain is usually restricted with respect to the range actually employed for the computation. On a workstation Pentium IV 2, $4 \mathrm{GHz}$ with 1 Gbyte of RAM, the computation of one shock wave structure took approximately $7 \mathrm{~h}$. 

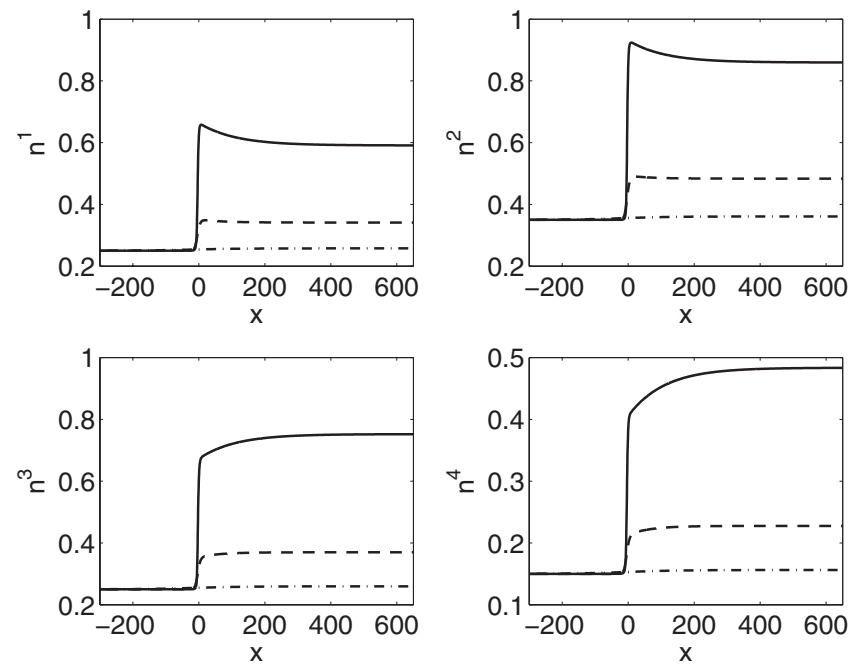

FIG. 1. Steady shock profile of the four number densities $n^{s}$ for problem A for three different choices of the concentration jump: $\Delta \chi=-0.03$ (solid line), $\Delta \chi=-0.01$ (dashed line), and $\Delta \chi=-0.001$ (dot-dashed line). The corresponding upstream Mach numbers are quoted in text [see Eq. (46)].

Profiles for number densities are shown in Fig. 1. The very smooth monotonic variation between only slightly different upstream and downstream limits occurring for small Mach numbers is hardly visible in the chosen scale. The profile steepens and the asymptotes separate for increasing Mach, and at a moderately high value of $\mathrm{Ma}_{-}$, we observe already a quite stiff front followed by a slower tail, as well as occurrence of overshooting for the two reactants. These phenomena are in agreement with previous simpler hydrodynamic-type calculations on traveling wave solutions, which are reliable enough when $\nu_{12}^{34}$ is small with respect to the corresponding mechanical quantities. In fact, in that frame, smooth solutions have been found, restricted to only quite small values of Mach number, ${ }^{17}$ however, at higher Ma_, a jump discontinuity develops in the wave front with upstream limit equal to the upstream asymptotic equilibrium, whereas a smooth tail joins the downstream limit to the downstream boundary condition. ${ }^{18}$ The discontinuity is replaced in our kinetic frame by strong but smooth transitions at higher and higher slope. The overshooting phenomenon seems not to appear instead for products, or for the total number density $n$, in agreement again with Ref. 18 . The ki-
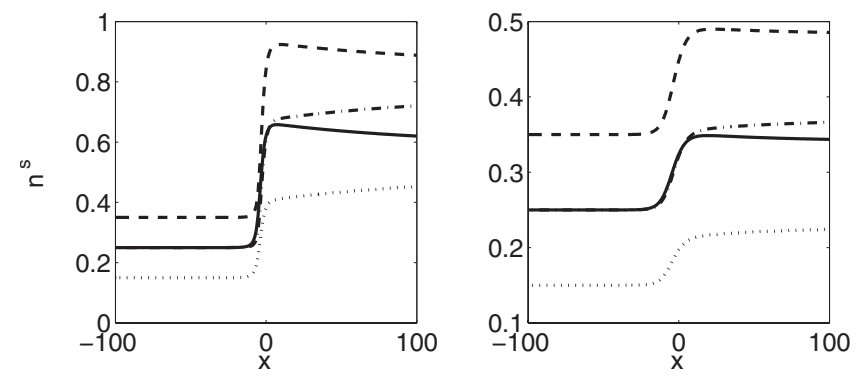

FIG. 2. Zoom of the steady shock profiles in Fig. 1 for $\Delta \chi=-0.03$ (left) and for $\Delta \chi=-0.01$ (right): species 1 (solid line), species 2 (dashed line), species 3 (dot-dashed line), and species 4 (dotted line).
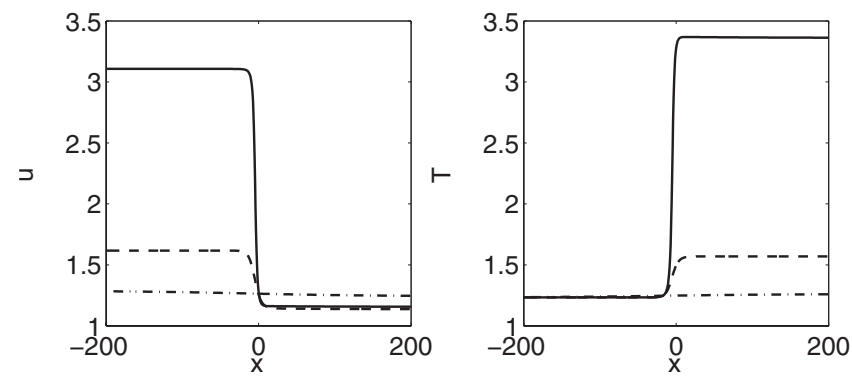

FIG. 3. Steady shock profile of the mass velocity $u$ and of the gas temperature $T$ for problem A for three different choices of the concentration jump: $\Delta \chi=-0.03$ (solid line), $\Delta \chi=-0.01$ (dashed line), and $\Delta \chi=-0.001$ (dotdashed line). The corresponding upstream Mach numbers are quoted in text [see Eq. (46)].

netic smooth structure of the four number densities occurring for the two cases with higher Mach number is better seen on the stretched scale in the two plots in Fig. 2.

Features similar to the ones described above are in order for the mass velocity $u$ and for the gas temperature $T$ reported in Fig. 3, but now overshooting is not present, nor are tails as long as before, illustrating the fact (properly captured by the present BGK approximation) that concentration fractions are the slowest in the relaxation process to downstream equilibrium, as a consequence of the weaker probability for a chemical reaction in a collision. This is confirmed by Figs. 4 and 5. In the former we plot, as a measure of the mechanical nonequilibrium along the whole $x$-axis, the deviations of species velocities $u^{s}$ and species temperatures $T^{s}$ with respect to their global values in the mixture, $u$ and $T$, respectively, when $\Delta \chi=-0.03$. The nonequilibrium region is quite small and changes only very slightly for varying species. The latter figure shows instead, as measure of chemical nonequilibrium, the quantity

$$
\Delta M=n^{1} n^{2}-\eta \exp (1 / T) n^{3} n^{4},
$$

which represents a sort of bias in the exothermic direction and vanishes, of course, when the mass action law is in or-
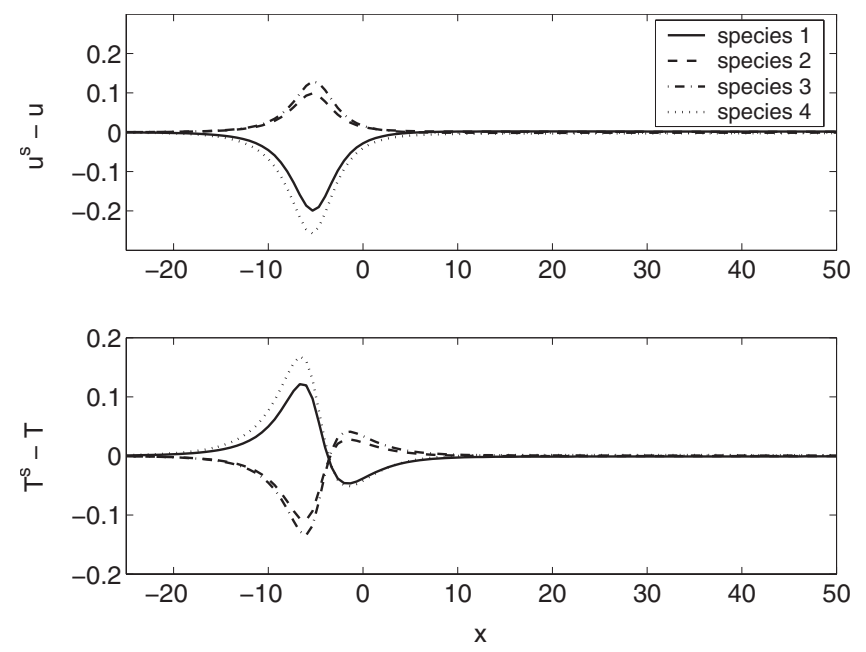

FIG. 4. Deviations of the species drift velocities $u^{s}$ and temperatures $T^{s}$ from global mass velocity $u$ and global temperature $T$ for problem A when $\Delta \chi=-0.03: s=1$ (solid line), $s=2$ (dashed line), $s=3$ (dot-dashed line), and $s=4$ (dotted line). 


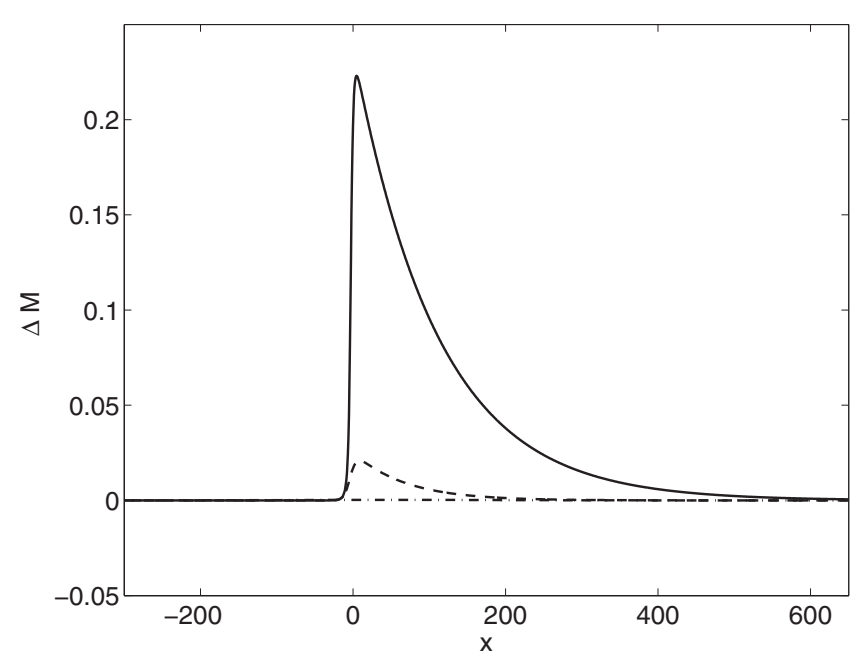

FIG. 5. Chemical nonequilibrium, quantified by Eq. (47), along the shock for problem A for three different choices of the concentration jump: $\Delta \chi$ $=-0.03$ (solid line), $\Delta \chi=-0.01$ (dashed line), and $\Delta \chi=-0.001$ (dot-dashed line). The corresponding upstream Mach numbers are quoted in text [see Eq. (46)].

der. Such quantity makes up, with opposite sign, part of the chemical source term (28), which, in turn, represents an estimate of the overall reaction rate for reactants. It is apparent how the reaction region, as well as the height of the peak, increases substantially for decreasing $\Delta \chi$ (i.e., for increasing $\mathrm{Ma}_{-}$), and may reach a considerable thickness, still preserving the feature of a sharp front upstream with a smooth tail downstream.

In order to illustrate the effects of different upstream conditions, we consider now a second case, to be labeled as problem $\mathrm{B}$, which differs from the previous one only in the equilibrium chemical composition at $-\infty$, taken now to be strongly unbalanced toward reactants
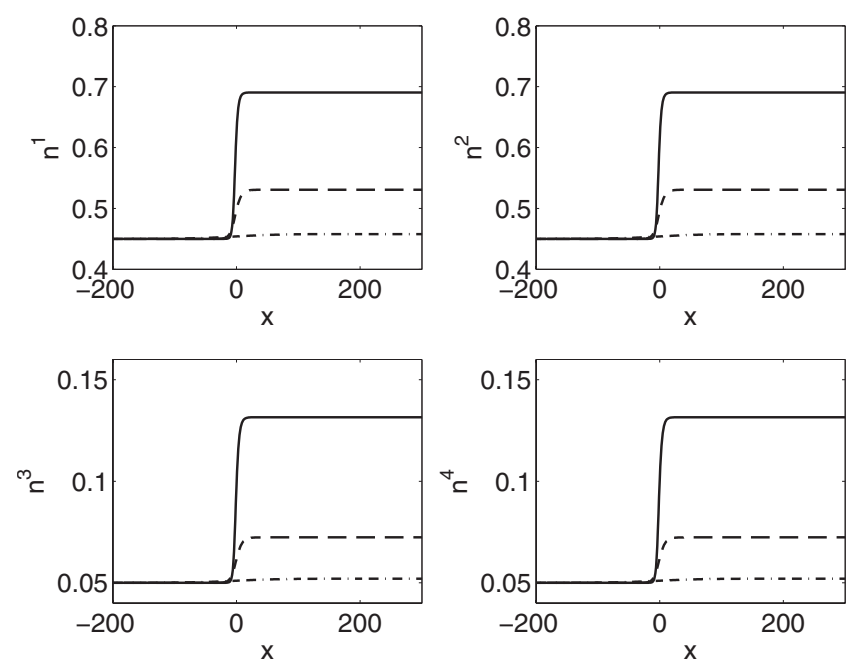

FIG. 6. Steady shock profile of the four number densities $n^{s}$ for problem B for three different choices of the concentration jump: $\Delta \chi=-0.03$ (solid line), $\Delta \chi=-0.01$ (dashed line), and $\Delta \chi=-0.001$ (dot-dashed line). The corresponding upstream Mach numbers are quoted in text [see Eq. (49)].

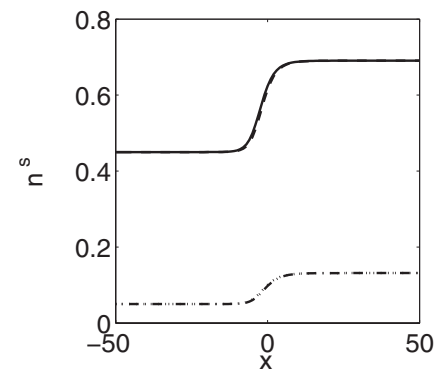

Phys. Fluids 20, 117103 (2008)

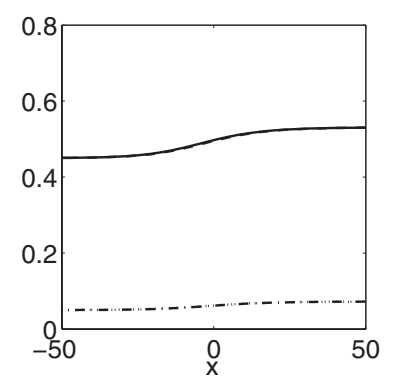

FIG. 7. Zoom of the steady shock profiles in Fig. 6 for $\Delta \chi=-0.03$ (left) and for $\Delta \chi=-0.01$ (right): species 1 (solid line), species 2 (dashed line), species 3 (dot-dashed line), and species 4 (dotted line).

$$
\chi_{-}^{1}=0.45, \quad \chi_{-}^{2}=0.45, \quad \chi_{-}^{3}=0.05, \quad \chi_{-}^{4}=0.05,
$$

implying $T_{-}=0.2295$. We keep the same values of $\Delta \chi$ associated now, of course, to different Mach numbers,

$$
\begin{aligned}
& \Delta \chi=-0.03,-0.01,-0.001 \\
& \left(\mathrm{Ma}_{-}=1.4109,1.1297,1.0125\right),
\end{aligned}
$$

and to different asymptotic boundary values,

$$
\begin{aligned}
& u_{-}=0.7500,0.6005,0.5382 \text {, } \\
& u_{+}=0.4562,0.4979,0.5278 \text {, } \\
& n_{+}=1.6441,1.2061,1.0198 \text {, } \\
& T_{+}=0.3049, \quad 0.2533,0.2318 .
\end{aligned}
$$

The general trends remain qualitatively similar to the previous case and, in fact, overshooting and extended tails have not yet appeared for the chosen values of $\mathrm{Ma}_{-}$, as shown by the density profiles in Fig. 6, which is the counterpart of Fig. 1 above. Smooth shock profiles replacing the jumps that would be in order at the hydrodynamic level are again emphasized in Fig. 7, structured in the same way as Fig. 2.

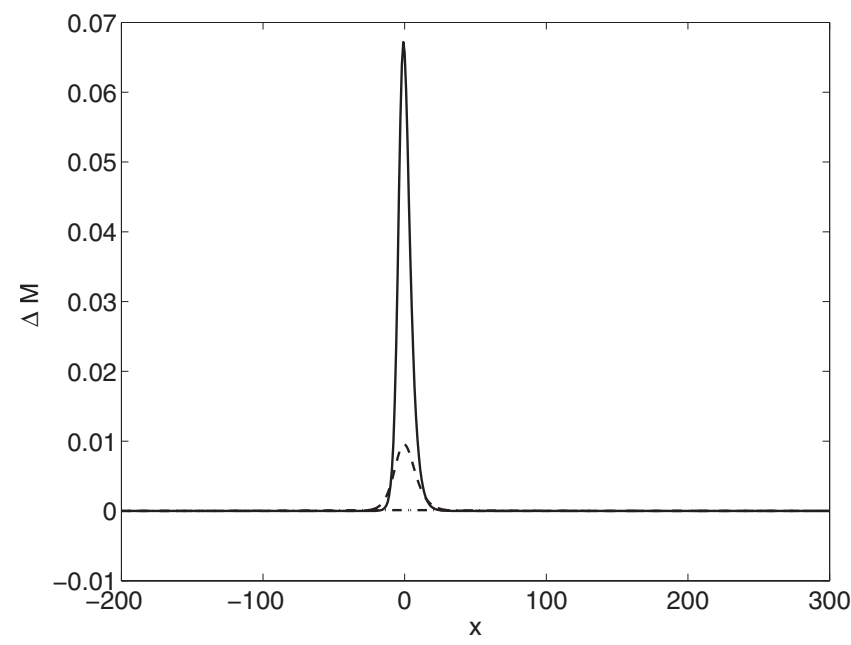

FIG. 8. Chemical nonequilibrium, quantified by Eq. (47), along the shock for problem B for three different choices of the concentration jump: $\Delta \chi$ $=-0.03$ (solid line), $\Delta \chi=-0.01$ (dashed line), and $\Delta \chi=-0.001$ (dot-dashed line). The corresponding upstream Mach numbers are quoted in text [see Eq. (49)]. 

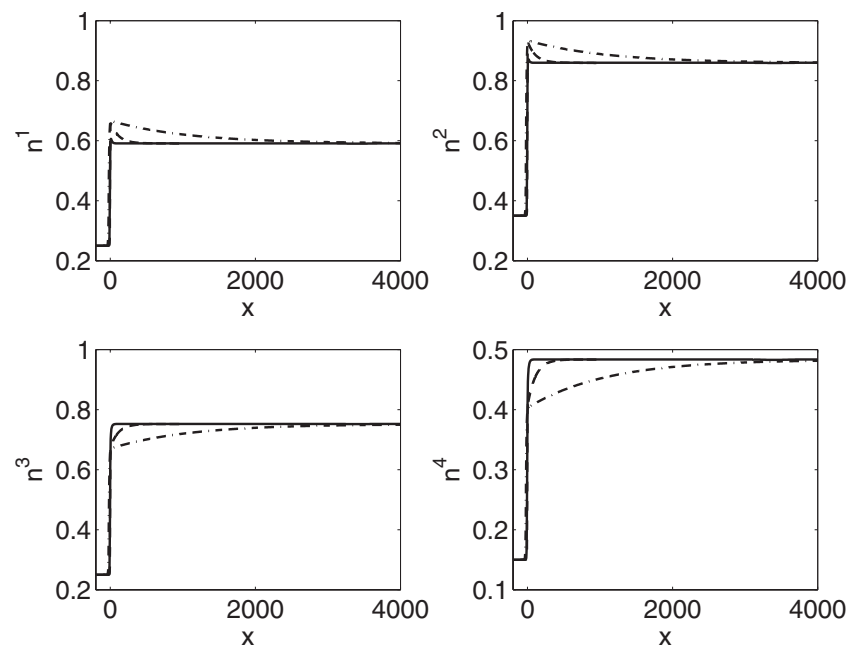

FIG. 9. Steady shock profile of the four number densities $n^{s}$ for test A when $\Delta \chi=-0.03$ for three different choices of the chemical collision frequency: $\nu_{12}^{34}=0.3$ (solid line), $\nu_{12}^{34}=0.03$ (dashed line), and $\nu_{12}^{34}=0.003$ (dot-dashed line).

Trends of the two reactants (products) are almost indistinguishable because of the symmetry in the upstream condition (48). Concerning the reaction region, Fig. 8 (to be compared to the previous Fig. 5) indicates that its width is not much affected by variation in parameters in the considered range but the intensity of the reactive burst increases substantially for decreasing $\Delta \chi$, as imposed by the underlying much stronger change in the chemical composition.

We pass then to consider the effects of the chemical collision frequency $\nu_{12}^{34}$ on the above picture, keeping all other parameters constant, in particular, the scattering collision frequencies, in order to illustrate the variations induced by a different relative importance of chemical reactions in the overall collision process. With reference to problem A, we now fix $\Delta \chi=-0.03$ and select three different values for the chemical collision frequency,

$$
\nu_{12}^{34}=0.3, \quad \nu_{12}^{34}=0.03, \quad \nu_{12}^{34}=0.003,
$$

where the first and the third ones are then one order of magnitude larger and smaller than the second, which is the reference value used in both previous tests A and B. For all three options, Mach number and other equilibrium boundary limits are obviously the same as in problem A. Upstream concentrations are provided by Eq. (45). Shock profiles for the single number densities are shown in Fig. 9 and should be compared again to the pertinent choice of $\Delta \chi$ in Fig. 1. As expected, at this relatively high Mach number (2.4698), the sharp shock front upstream is very scarcely influenced by the different chemical characteristic times, which instead affects dramatically the relaxation tail downstream that almost disappears for the fastest reaction but conversely becomes extremely long and slow for the slowest one. In Fig. 10 we report also for completeness a related plot relevant to problem B with the same options for $\nu_{12}^{34}$. Now composition upstream is given by Eq. (48), and we fix $\Delta \chi=-0.001$, implying Ma_=1.0125. This plot should be compared to one of the options in Fig. 6, where however the very regular and almost symmetric trend of the shock profiles is hardly visible on the
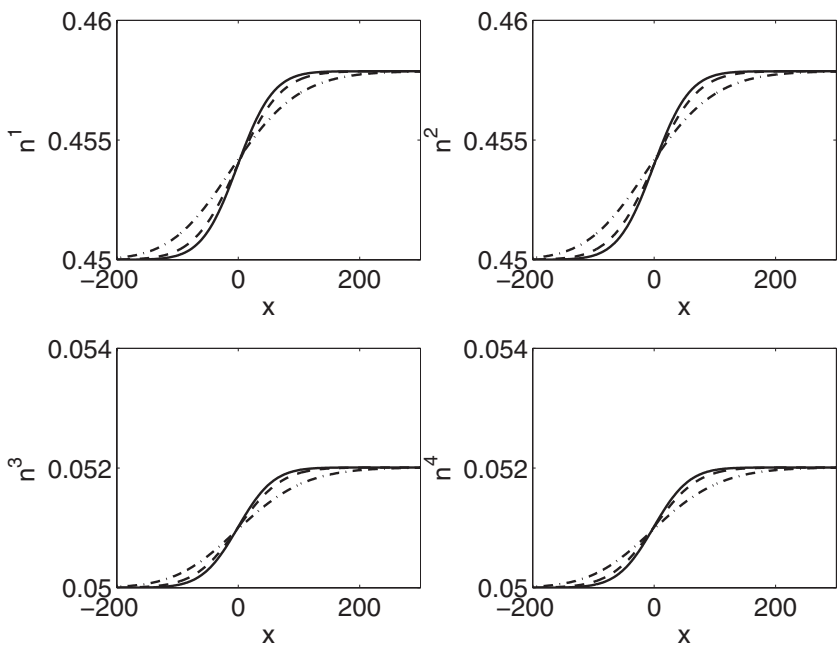

FIG. 10. Steady shock profile of the four number densities $n^{s}$ for test B when $\Delta \chi=-0.001$ for three different choices of the chemical collision frequency: $\nu_{12}^{34}=0.3$ (solid line), $\nu_{12}^{34}=0.03$ (dashed line), and $\nu_{12}^{34}=0.003$ (dotdashed line).

much more compressed scale. It is again evident that increasing $\nu_{12}^{34}$ amounts to reducing the shock thickness and enhancing the approach to the asymptotic upstream and downstream equilibria. All of the above features can be cross-checked in the four plots of Fig. 11, where the quantity $\Delta M$, proportional to the chemical source term for products, is reported for the three selected values of $\nu_{12}^{34}$ for both tests $\mathrm{A}$ and $\mathrm{B}$, with reference to both $\Delta \chi=-0.03$ and $\Delta \chi=-0.001$ cases. Notice the difference by order of magnitudes between the horizontal scales of the two rows and, similarly, between the vertical scales of the two columns. The Mach numbers relevant to the four plots in their natural order are 2.4698, 1.0227, 1.4109, and 1.0125. In making comparisons among reaction rates, one should of course bear in mind the multiplicative factor $\nu_{12}^{34}$ that is present in Eq. (28), which en-
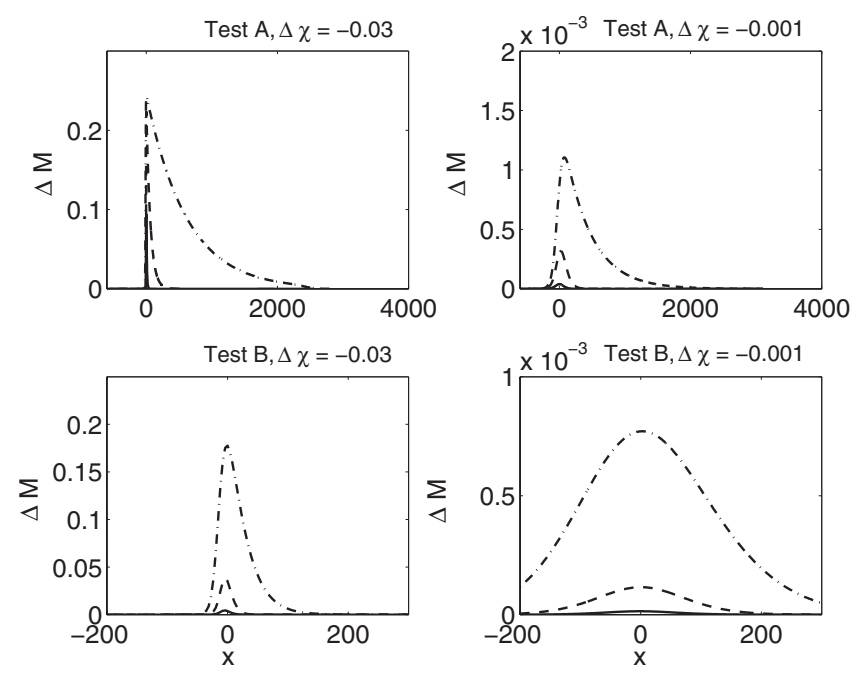

FIG. 11. Chemical nonequilibrium, quantified by Eq. (47), along the shock for both tests A and B with either $\Delta \chi=-0.03$ or $\Delta \chi=-0.001$ for three different choices of the chemical collision frequency: $\nu_{12}^{34}=0.3$ (solid line), $\nu_{12}^{34}=0.03$ (dashed line), and $\nu_{12}^{34}=0.003$ (dot-dashed line). 
hances or reduces the ratios among the quantities (47) shown by the figure.

\section{CONCLUDING REMARKS}

The steady shock problem in one space dimension for a mixture of rarefied gases undergoing a reversible bimolecular chemical reaction is studied and numerically solved at a kinetic level. A consistent relaxation-time approximation of BGK type, which is suitable for moderately slow (in comparison to mechanical scattering) chemical processes, has been employed. The numerical solution has been obtained by an appropriate iterative scheme based on upwind finite difference approximation. Results for the profiles of the main macroscopic observables (densities, velocities, temperatures, and reaction rates) have been illustrated and discussed for several choices of parameters, essentially the Mach number, upstream chemical composition, and reactive collision frequency.

The main features expected from a physical point of view are well described by the proposed model. In particular, the smooth profiles occurring for small Mach numbers steepen for increasing Mach to produce eventually stiff fronts, followed by a slower tail downstream, with possible occurrence, at moderately high Mach numbers, of overshooting in some of the densities. This effect turns out to be the stronger, the smaller is the chemical collision frequency. The different relaxation processes (mechanical and chemical) can be seen in the plots, and the latter is, of course, slower for the parameter values to which the BGK model applies. Reaction region and shock thickness may increase substantially for increasing chemical relaxation time. Results compare well with the previous calculations based on a hydrodynamic limit for vanishing mechanical Knudsen number of the reactive Boltzmann equations, both at low and at high Mach numbers. Of course, in such a limit, the stiff, but still smooth, front is replaced by a jump discontinuity. A systematic quantitative analysis and comparison is in progress, as well as the employment for the shock problem of a different BGK model, suitable when mechanical and chemical relaxations are of the same order. It would be nice to compare quantitatively the present results to reactive DSMC or deterministic kinetic calculations, which however the authors are not aware of. The latter schemes will hopefully be a matter of future investigation. Also scheduled as future work is the extension of the present analysis to more than one space dimension, a nontrivial problem with its own technical difficulties (especially from a numerical point of view), that would be of significant interest toward the application of kinetic equations for solving classical problems.

\section{ACKNOWLEDGMENTS}

This work was performed in the frame of the activities sponsored by MIUR (Project "Nonconservative binary interactions in various types of kinetic models"), by INdAM, by GNFM, and by the University of Parma (Italy). It was also partially supported by the Grant-in-Aid for Scientific Research from JSPS (Grant No. 20360046) (Japan) and by DAAD (Germany). The authors are grateful to Professor Shigeru Takata for valuable discussions.

${ }^{1}$ I. Prigogine and E. Xhrouet, "On the perturbation of Maxwell distribution function by chemical reaction in gases," Physica (Amsterdam) 15, 913 (1949).

${ }^{2}$ C. Cercignani, The Boltzmann Equation and Its Applications (Springer, New York, 1988).

${ }^{3}$ L. Sirovich, "Kinetic modeling of gas mixtures," Phys. Fluids 5, 908 (1962).

${ }^{4}$ V. Garzó, A. Santos, and J. J. Brey, "A kinetic model for a multicomponent gas," Phys. Fluids A 1, 380 (1989).

${ }^{5}$ V. Giovangigli, Multicomponent Flow Modeling (Birkhäuser, Boston, 1999).

${ }^{6} \mathrm{M}$. Groppi and G. Spiga, "Kinetic approach to chemical reactions and inelastic transitions in a rarefied gas," J. Math. Chem. 26, 197 (1999).

${ }^{7}$ L. Desvillettes, R. Monaco, and F. Salvarani, "A kinetic model allowing to obtain the energy law of polytropic gases in the presence of chemical reactions," Eur. J. Mech. B/Fluids 24, 219 (2005).

${ }^{8}$ R. Monaco and M. Pandolfi Bianchi, "A BGK type model for a gas mixture with reversible reactions," in New Trends in Mathematical Physics (World Scientific, Singapore, 2004), pp. 107-120.

${ }^{9}$ M. Groppi and G. Spiga, "A Bhatnagar-Gross-Krook-type approach for chemically reacting gas mixtures," Phys. Fluids 16, 4273 (2004).

${ }^{10}$ G. M. Kremer, M. Pandolfi Bianchi, and A. J. Soares, "A relaxation kinetic model for transport phenomena in a reactive flow," Phys. Fluids 18, 037104 (2006).

${ }^{11}$ Y. Sone, Kinetic Theory and Fluid Dynamics (Birkhäuser, Boston, 2002).

${ }^{12} \mathrm{~T}$. Ohwada, "Structure of normal shock waves: Direct numerical analysis of the Boltzmann equation for hard-sphere molecules," Phys. Fluids A 5, 217 (1993).

${ }^{13}$ F. Filbet and G. Russo, "High order numerical methods for the space non-homogeneous Boltzmann equation,” J. Comput. Phys. 186, 457 (2003).

${ }^{14}$ S. Kosuge, K. Aoki, and S. Takata, "Shock-wave structure for a binary gas mixture: Finite-difference analysis of the Boltzmann equation for hardsphere molecules," Eur. J. Mech. B/Fluids 20, 87 (2001).

${ }^{15}$ Y. Sone, Molecular Gas Dynamics: Theory, Techniques, and Applications (Birkhäuser, Boston, 2007).

${ }^{16}$ F. Conforto, R. Monaco, F. Schürrer, and I. Ziegler, "Steady detonation waves via the Boltzmann equation for a reacting mixture," J. Phys. A 36, 5381 (2003).

${ }^{17}$ A. Rossani and A. M. Scarfone, "Travelling waves in a mixture of gases with bimolecular reversible reactions," Physica B 348, 198 (2004).

${ }^{18}$ F. Conforto, M. Groppi, R. Monaco, and G. Spiga, "Steady detonation problem for slow and fast chemical reaction," in Modelling and Numerics of Kinetic Dissipative Systems, edited by L. Pareschi, G. Russo, and G. Toscani (Nova Science, New York, 2006), pp. 105-117.

${ }^{19}$ M. Groppi, P. Lichtenberger, F. Schürrer, and G. Spiga, "Conservative approximation schemes of kinetic equations for chemical reactions," Eur. J. Mech. B/Fluids 27, 202 (2008).

${ }^{20}$ A. Aimi, M. Diligenti, M. Groppi, and C. Guardasoni, "On the numerical solution of a BGK-type model for chemical reactions," Eur. J. Mech. B/Fluids 26, 455 (2007).

${ }^{21}$ Ya. B. Zel'dovich and Yu. P. Raizer, Physics of Shock Waves and HighTemperature Hydrodynamic Phenomena (Dover, Mineola, NY, 2002).

${ }^{22}$ G. Boillat and T. Ruggeri, "On the shock structure problem for hyperbolic system of balance laws and convex entropy," Continuum Mech. Thermodyn. 10, 285 (1998).

${ }^{23}$ T. Ruggeri, "Global existence, stability and non linear wave propagation in binary mixtures of Euler fluids," in New Trends in Mathematical Physics (World Scientific, Singapore, 2004), pp. 205-214.

${ }^{24}$ A. Rossani and G. Spiga, "A note on the kinetic theory of chemically reacting gases," Physica A 272, 563 (1999). 
${ }^{25}$ M. Bisi, M. Groppi, and G. Spiga, "A kinetic model for bimolecular chemical reactions," in Kinetic Methods for Nonconservative and Reacting Systems, Quaderni di Matematica Vol. 16, edited by G. Toscani (Seconda Università di Napoli, Caserta. Aracne Editrice S.r.l., Roma, 2005), pp. $1-145$.

${ }^{26}$ M. Groppi, G. Spiga, and S. Takata, "The steady shock problem in reactive gas mixtures," Bullet. Inst. Math. Acad. Sinica (New Series) 2, 935 (2007).

${ }^{27}$ P. Andries, K. Aoki, and B. Perthame, "A consistent BGK-type model for gas mixtures," J. Stat. Phys. 106, 993 (2002).

${ }^{28}$ M. Bisi, M. Groppi, and G. Spiga, "Grad's distribution functions in the kinetic equations for a chemical reaction," Continuum Mech. Thermodyn. 14, 207 (2002).

${ }^{29}$ Handbook of Mathematical Functions, edited by M. Abramowitz and I. A. Stegun (Dover, New York, 1965).

${ }^{30} \mathrm{C}$. K. Chu, "Kinetic-theoretic description of the formation of a shock wave," Phys. Fluids 8, 12 (1965).

${ }^{31}$ K. Aoki, Y. Sone, and T. Yamada, "Numerical analysis of gas flows condensing on its plane condensed phase on the basis of kinetic theory," Phys. Fluids A 2, 1867 (1990).

${ }^{32} \mathrm{~V}$. Tritsch, "Shock waves in four component chemically reacting gases," Ph.D. thesis, Johannes-Gutenberg University, 2007. 